\title{
TROMBOEMBOLIA PULMONAR APÓS VIDEOARTROSCOPIA DE OMBRO
}

\section{THROMBOEMBOLIC COMPLICATION AFTER ARTHROSCOPIC SHOULDER SURGERY}

Fabio Farina Dal Molin', Siluê Franzoni Dal Molin²

\section{RESUMO}

Embora fenômenos tromboembólicos sejam complicações frequentes em cirurgias dos membros inferiores, apenas dois relatos de casos de tromboembolia pulmonar após artroscopia de ombro são encontrados na literatura. É descrito o caso de uma paciente com 76 anos com embolia pulmonar bilateral após artroscopia cirúrgica do ombro. Não foram encontradas anormalidades vasculares e nenhuma origem do trombo foi detectada, ficando desconhecida a causa exata responsável pela tromboembolia.

Descritores - Artroscopia; Ombro; Embolia pulmonar

\section{ABSTRACT}

Though thromboembolisms are frequent complications of surgery of the lower extremities, only two cases of pulmonary thromboembolism after shoulder arthroscopy are found in the literature. We describe the case of a 76-year-old patient with pulmonary embolism in both lungs after shoulder arthroscopy. No vascular abnormalities nor the origin of the thrombus was detected. The etiology of the thromboembolism remains unknown.

Keywords - Arthroscopy; Shoulder; Pulmonary embolism

\section{INTRODUÇÃO}

A trombose venosa profunda (TVP) e a embolia pulmonar (EP) são complicações conhecidas das cirurgias ortopédicas dos membros inferiores. Nas artroplastias de joelho e quadril sem profilaxia, os fenômenos tromboembólicos apresentam incidência de $29 \%$ a $60 \%{ }^{(1,2)}$. Nas cirurgias artroscópicas do joelho, encontra-se incidência de TVP em até $4 \%{ }^{(3)}$, sendo que a EP sintomática está presente em menos de $1 \%$ dos pacientes ${ }^{(4)}$. Embora existam relatos de tromboembolia pulmonar fatal após artroplastia de ombro ${ }^{(5)}$, esta complicação é pouco frequente após videoartroscopia desta articulação.

O objetivo deste trabalho é relatar o caso de uma paciente que desenvolveu tromboembolia pulmonar bilateral após videoartroscopia de ombro.

\section{RELATO DE CASO}

Paciente NMF, 76 anos, sexo feminino, do lar, apresentava queixa de dor em ombro esquerdo havia um ano.
A dor era como agulhadas, principalmente ao levantar o braço ou em movimentos ocasionais e inesperados. A paciente apresentava diabetes tipo II, hipertensão arterial sistêmica, fazia reposição hormonal e estava acima de seu peso (70kg, 1,60m).

Ao exame físico apresentava mobilidade do ombro direito e esquerdo simétrica (150, 45, T 9), força de abdução quatro, força de rotação interna e externa cinco, Speed +, Jobe + Neer + . Ao elevar o braço, fazia um movimento específico para evitar a dor.

No exame radiográfico do ombro apresentava acrômio tipo II e cistos subcondrais na região do sulco intertubercular do úmero. Na ressonância nuclear magnética (RNM) (Figuras 1 e 2), apresentava líquido subacromial aumentado, lesão do supraespinhal com $2 \mathrm{~cm}$ de retração, tendão do bíceps alargado com lesões intramurais e artrose acromioclavicular.

Nos exames pré-operatórios, a paciente não apresentava anormalidades sanguíneas, função renal preservada e sem hipercolesterolemia.

1 - Mestre em Ortopedia e Traumatologia pela FCMSCSP, Ortopedista Especialista em Ombro e Cotovelo do Hospital Moinhos de Vento, Porto Alegre, RS.

2 - Mestre em Anestesiologia pela UFRGS, Anestesista do Hospital de Clínicas de Porto Alegre e Hospital Moinhos de Vento, Porto Alegre, RS.

Trabalho realizado no Hospital Moinhos de Vento, Porto Alegre, RS.

Correspondência: Av. Mariland, 1.314. ap. 602 - Mont Serrat - 90440-190 - Porto Alegre, RS - E-mail: dalmolin@ombrocotovelo.com.br 


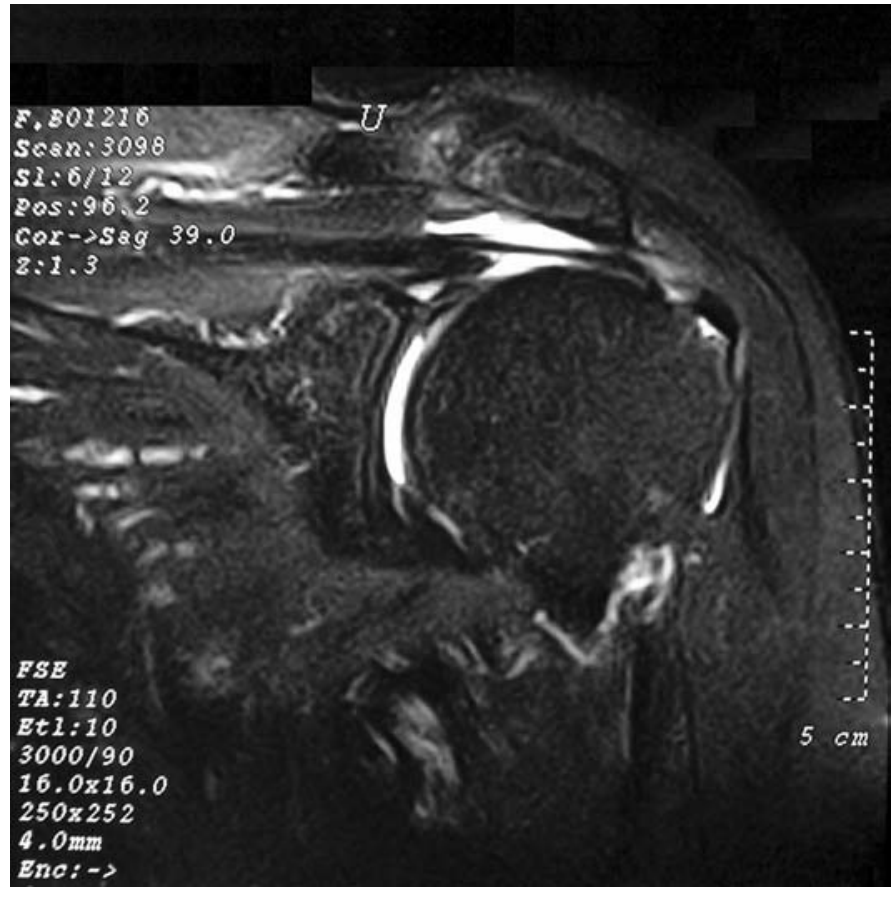

Figura 1 - RNM do ombro esquerdo em corte coronal, mostrando lesão do tendão do músculo supraespinhal

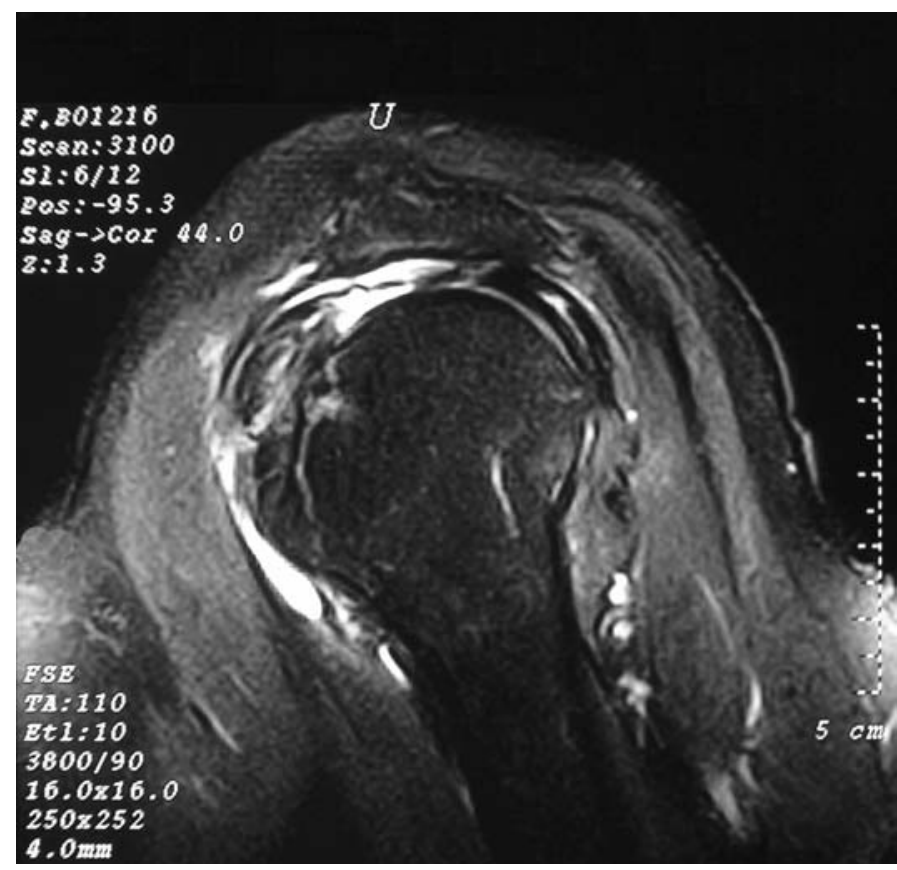

Figura 2 - RNM do ombro esquerdo em corte sagital, mostrando lesão do tendão do músculo supraespinhal e tendão da cabeça longa do bíceps do braço alargado

Diabetes, colesterol e pressão arterial estavam controlados com medicação. Fazia reposição hormonal. Negava tabagismo ou fenômenos alérgicos. Não fazia uso de anticoagulantes.

A paciente foi submetida a videoartroscopia de ombro para sutura do manguito rotador e tenotomia do cabo longo do bíceps. A mesma submeteu-se a bloqueio interescalênico e anestesia geral. Foi posicionada em cadeira de praia com 70 graus de flexão do tronco e permaneceu nesta posição por 2,5 horas. O braço direito permaneceu em abdução de 70 graus e paralelo ao tronco. O braço esquerdo foi deixado rente ao corpo, apoiado em um suporte.

A inspeção artroscópica mostrou um tendão do bíceps degenerado e alargado. Havia líquido sinovial no espaço subacromial e o manguito rotador apresentava lesão do supraespinhal em U, com 2cm de retração (Figura 3). O bíceps foi tenodesado, foi feita acromioplastia e ressecção da extremidade lateral da clavícula, pois houve lesão da estrutura capsuloligamentar inferior da articulação acromioclavicular e a mesma apresentava-se com artrose avançada. A lesão em U do supraespinhal foi suturada com um ponto tendão-tendão e duas âncoras, segundo a técnica de dupla fileira: foi colocada uma âncora com dois fios no colo anatômico e feito dois pontos tipo matress. Uma segunda âncora com um fio foi colocada no tubérculo maior e feito um ponto em $\mathrm{U}$, passando pelas bordas anterior e posterior da lesão, fazendo o fechamento completo desta (Figura 4). A bomba de infusão foi utilizada em 40mmHg e, ocasionalmente, em $60 \mathrm{mmHg}$, para controlar e realizar a hemostasia com radiofrequência.

Na sala de recuperação, uma hora após o procedimento, a paciente apresentava-se ansiosa, impaciente, queixando-se de desconforto inespecífico na região

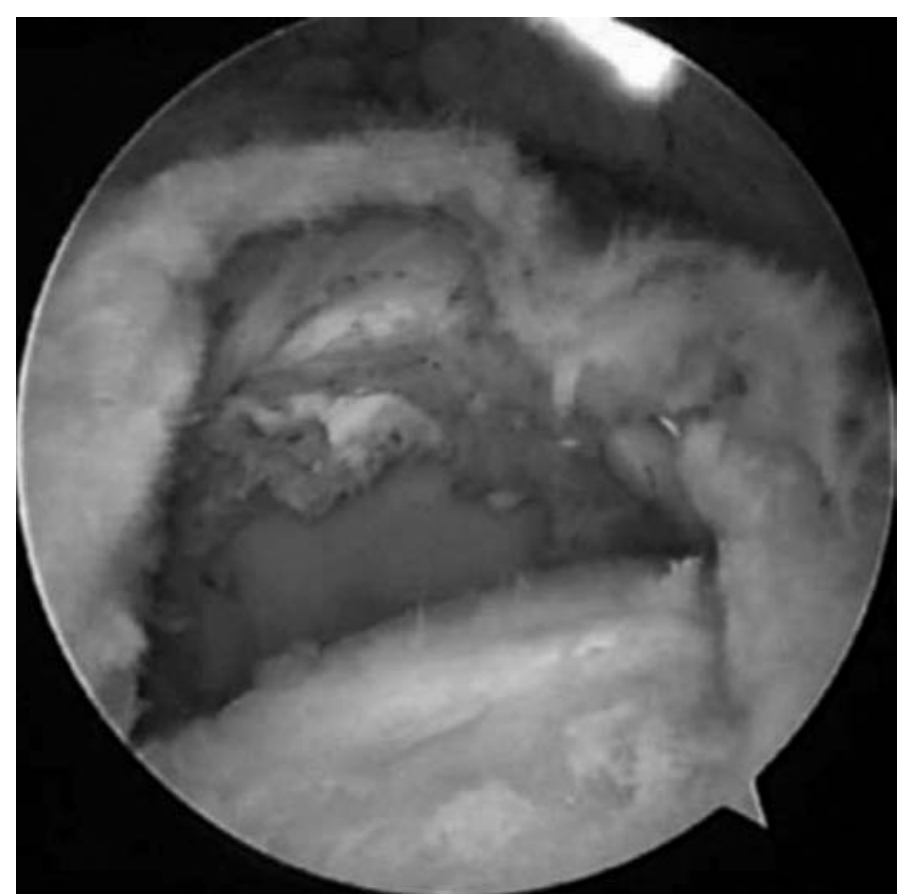

Figura 3 - Lesão do tendão do músculo supraespinhal em U 


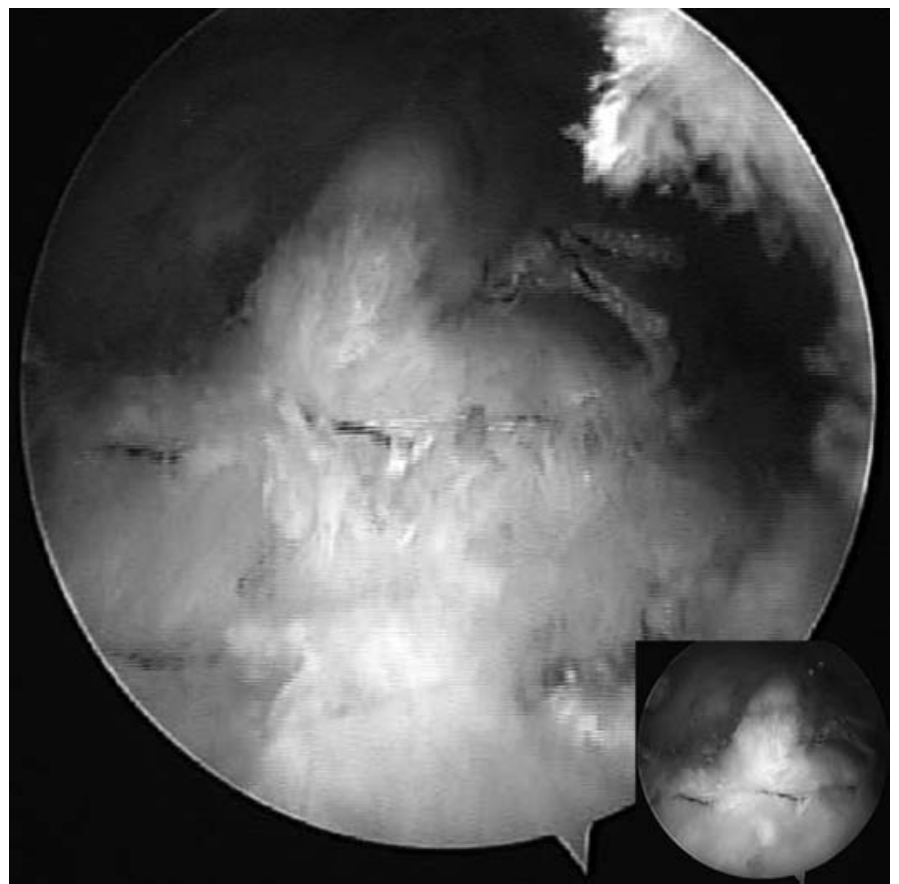

Figura 4 - Lesão do tendão do músculo supraespinhal suturada com dupla fileira. Imagem menor mostrando um ponto tendãotendão e a fileira medial. Imagem maior mostrando também a fileira lateral

esofagiana alta. Queixava-se também de dor no ombro esquerdo, apesar de estar sob efeito analgésico de bloqueio interescalênico. Como a queixa de dor era importante, a paciente foi tratada com sulfato de morfina, mas não apresentou melhora nos sintomas. A saturação arterial de oxigênio $\left(\mathrm{SpO}_{2}\right)$ com cateter nasal permanecia em 94-96\%. Nas horas seguintes, a paciente apresentava-se mais queixosa, gemente, e a saturação diminuiu para 93-94\%. O oxigênio foi aumentado para $5 \mathrm{~L} / \mathrm{min}$ e, três horas após o procedimento, a $\mathrm{SpO}_{2}$ estava em $92 \%$. A paciente foi reavaliada e referia rouquidão e desconforto retroesternal. A ausculta pulmonar apresentava estertores crepitantes bilaterais e foi administrado diurético para tratamento de provável edema agudo de pulmão. Solicitou-se radiografia de tórax, a qual apresentou consolidação na base pulmonar direita. A gasometria arterial apresentou $\mathrm{SpO}_{2}$ de $90 \%$ e D-Dímeros entre 500-1.000ng/mL (valor de referência $<250 \mathrm{ng} / \mathrm{mL}$ ). A paciente permaneceu com oxigênio $3 \mathrm{~L} / \mathrm{min}$ por mais dois dias e a saturação sanguínea de oxigênio permaneceu em torno de 94-95\%. Pressão arterial e glicemia estavam controladas. A tomografia computadorizada de tórax apresentou algumas opacidades centrolobulares e estrias atelectásicas no lobo inferior esquerdo de etiologia inflamatória/pós-inflamatória.
A ecocardiografia com Doppler apresentou quadro de mínima insuficiência mitral e tricúspide, e permitia estimar a pressão pulmonar em 41mmHg. A cintilografia perfusional pulmonar (Figura 5) encontrou evidências de várias áreas hipoperfundidas anormais subsegmentares nos dois pulmões, altamente sugestivo de embolia pulmonar. A ecodoppler do sistema venoso dos membros inferiores não apresentou sinais de trombose ou refluxo no sistema venoso profundo dos membros inferiores.

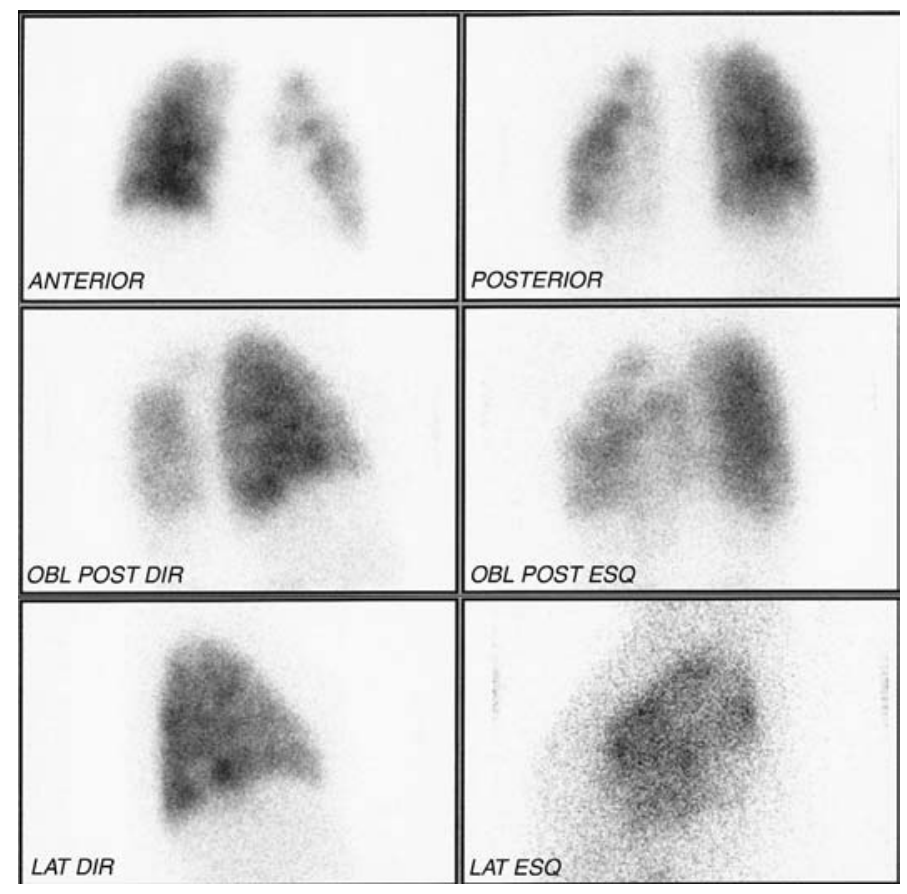

Figura 5 - Cintilografia perfusional pulmonar evidencia várias áreas hipoperfundidas anormais subsegmentares nos dois pulmões

\section{DISCUSSÃO}

Os fenômenos tromboembólicos são complicações frequentes em pacientes submetidos a cirurgias ortopédicas em membros inferiores. Patologia venosa tromboembólica após artroplastia de joelho e quadril ocorre entre $29 \%$ e $60 \%$ dos pacientes que não fazem profilaxia $^{(6)}$. Estudos de pacientes que realizaram artroscopia de joelho mostram que a incidência pode variar de $4 \%$ a $17 \%{ }^{(3,4,7)}$. A incidência desta patologia grave após cirurgia do membro superior não está bem definida. Lyman et al ${ }^{(6)}$, em estudo de prognóstico retrospectivo, encontraram incidência de 0,5\% de TVP após artroplastias de ombro. Após videoartroscopia de ombro, existem poucos casos relatados na literatura ${ }^{(8,9)}$. 
Existe associação clara entre trombose venosa profunda e embolia pulmonar. A incidência de tromboembolia pulmonar aumenta com a incidência de TVP. A tríade de Wirchow: hipercoagulabilidade, estase e lesão da íntima é a causa da formação do trombo. Estes fatores estão presentes tanto nas cirurgias dos membros inferiores como superiores ${ }^{(5)}$.

Os seguintes fatores têm sido associados com fenômenos tromboembólicos: idade, obesidade, trauma, deficiência de antitrombina III, deficiência de proteína C e S, câncer, uso de anticoncepcionais orais, infarto do miocárdio, imobilização prolongada, história prévia de EP ou TVP ${ }^{(5,6)}$.

Ao contrário do que na articulação do joelho e quadril, são pouco frequentes os estudos sistematizados que investiguem a incidência de TVP e EP no ombro. Somente alguns casos de complicações tromboembólicas após videoartroscopia de ombro estão relatados ${ }^{(8,9)}$. Relatos de TVP depois de artroscopia do membro superior correlacionam a TVP com coagulopatia e anormalidades anatômicas $^{(8,9)}$. No caso anteriormente descrito, a paciente apresentava vários fatores de risco para tromboembolia: idade avançada, diabética tipo II, uso de hormônios e obesidade. Neste caso, não encontramos nenhuma coagulopatia ou causa mecânica para a embolia. Assim, a verdadeira causa da embolia pulmonar do caso que relatamos não foi encontrada. Somente podemos considerar a presença dos fatores de risco.

Polzhofer et al $^{(10)}$ acreditam que a lesão da veia subclávia, devido à compressão da ponteira do shaver, pode levar a fenômenos tromboembólicos, e referem que esta é a provável causa da patologia. Consideram que a po- sição inadequada do braço, a compressão causada pelo edema operatório e a tração elevada também podem contribuir para lesões da subclávia que levaria ao tromboembolismo. Em nosso caso, apesar de não usarmos tração do braço em cadeira de praia, concordamos que o edema causado pela infusão de soro pela bomba de infusão e a manipulação do shaver no portal anterior durante a ressecção da articulação acromioclavicular pode contribuir com a lesão da subclávia. Achamos também que a posição em 70 graus de flexão do tronco para a posição em cadeira de praia pode levar a estase e, consequentemente, tromboembolia. Também levantamos a hipótese de que a lesão mecânica do acrômio e clavícula durante a ressecção com burr, pode levar à embolia gordurosa.

O uso de profilaxia medicamentosa é controverso. Wirth et $a l^{(3)}$, em estudo controlado com heparina de baixo peso molecular, em 239 pacientes avaliados, seis apresentaram TVP, cinco no grupo controle e um no grupo tratado. Como a literatura atual não apresenta a incidência exata de complicação tromboembólicas, não há consenso sobre a recomendação de profilaxia com medicamentos. No entanto, concordamos com Polzhofer et $a l^{(10)}$ que a profilaxia com heparina de baixo peso molecular pode ser usada em pacientes de alto risco para complicações tromboembólicas.

\section{CONCLUSÃO}

A videoartroscopia cirúrgica do ombro é um procedimento seguro. Mas, embora sejam raras, complicações graves como embolia pulmonar podem ocorrer e devem sempre ser lembradas.

\section{REFERÊNCIAS}

1. Aaron RK, Ciombor D. Venous thromboembolism in the orthopedic patient. Surg Clin North Am. 1983;63(3):529-37.

2. Warwick D, Martin AG, Glew D, Bannister GC. Measurement of femoral vein blood flow during total hip replacement. Duplex ultrasound imaging with and without the use of a foot pump. J Bone Joint Surg Br. 1994;76(6):918-21.

3. Wirth T, Schneider B, Misselwitz F, Lomb M, Tüylü H, Egbring R, Griss P. Prevention of venous thromboembolism after knee arthroscopy with low-molecular weight heparin (reviparin): Results of a randomized controlled trial. Arthroscopy. 2001;17(4):393-9.

4. Schippinger G, Wirnsberger GH, Obernosterer A, Babinski K. Thromboembolic complications after arthroscopic knee surgery. Incidence and risk factors in 101 patients. Acta Orthop Scand. 1998;69(2):144-6.

5. Saleem A, Markel DC. Fatal pulmonary embolus after shoulder arthroplasty. J Arthroplasty. 2001;16(3):400-3.

6. Lyman S, Sherman S, Carter TI, Bach PB, MandI LA, Marx RG. Prevalence and risk factors for symptomatic thromboembolic events after shoulder arthroplasty. Clin Orthop Relat Res. 2006;448:152-6.

7. Demers C, Marcoux S, Ginsberg JS, Laroche F, Cloutier R, Poulin J. Incidence of venographically proved deep vein thrombosis after knee arthroscopy. Arch Intern Med. 1998;158(1):47-50.

8. Burkhart SS. Deep venous thrombosis after shoulder arthroscopy. Arthroscopy. 1990;6(1):61-3.

9. McFarland EG, O'Neill OR, Hsu CY. Complications of shoulder arthroscopy. J South Orthop Assoc. 1997;6(3):190-6.

10. Polzhofer GK, Petersen W, Hassenpflug J. Thromboembolic complication after arthroscopic shoulder surgery. Arthroscopy. 2003;19(9):E129-32. 